\title{
Characteristics of micronozzle gas flows
}

\author{
Chong Xie ${ }^{\mathrm{a})}$ \\ Key Laboratory of High Temperature Gas Dynamics, Institute of Mechanics of Chinese Academy \\ of Sciences, 15, North 4th Ring West Road, Beijing 100080, China
}

(Received 6 June 2006; accepted 23 January 2007; published online 27 March 2007)

\begin{abstract}
The fluid characteristics of gas flows in the micronozzle whose throat height is $20 \mu \mathrm{m}$ were investigated by the direct simulation Monte Carlo (DSMC) method. In a series of cases, the dependence of mass flux on the pressure difference was gained, and the DSMC's results show good agreement with the experimental data. The comparison of mass flux and the Mach number contours between the DSMC and Navier-Stokes equations adding slip boundary also reveals quantitatively that the continuum model will be invalid gradually even when the average Knudsen number is smaller than 0.01. As one focus of the present paper, the phenomenon of the multiple expansion-compression waves that comes from the nozzle's divergent part was analyzed in detailed. () 2007 American Institute of Physics. [DOI: 10.1063/1.2709707]
\end{abstract}

\section{INTRODUCTION}

With the rapid development of microelectromechanical systems (MEMS), many novel physical phenomena have appeared and interested researchers from extensive fields. As an extension, the study of gas flows in the microdimensional devices has gained many results. It has been acknowledged that a decrease of the characteristic length of the microsystem leads to a rapid increase of the surface-to-volume ratio, so the surface effect becomes prominent in the MEMS. ${ }^{1}$ For instance, the friction that comes from the wall may affect relatively more of the whole flow field. In addition, if the nondimensional Knudsen number $\mathrm{Kn}=\lambda / H$ (where the $\lambda$ is the mean free path of gas molecular and $H$ is the characteristic length of flows) is not a very small value $(\mathrm{Kn} \ll 1)$, the rarefied gas effect will appear gradually, and the continuum models will break down gradually too. It is accepted that the partition of the fluid regime can be based on the Knudsen number: ${ }^{2}$ when $\mathrm{Kn}>10$, the flow is called free molecular flow; when $10>\mathrm{Kn}>0.1$, the flow is in the range of the transition regime; if $0.1>\mathrm{Kn}>0.01$, the flow is in the slip regime; and when $0.01>\mathrm{Kn}$, the flow is in the continuum regime. It is generally believed that the atomistic nature of fluid may be ignored in the continuum regime. Certainly, the above limits are not fixed values, and they can be viewed as the reference of order. ${ }^{3,4}$ It is also known that the NavierStokes (NS) equations adding a slip boundary condition are valid in the slip regime. Some straight microchannel gas flows do verify that the NS equations well predict the experimental mass flux when $\mathrm{Kn}<0.1$ (even about $\mathrm{Kn}<0.3$ with a two-order slip boundary condition $\left.{ }^{5,6}\right)$, but a detailed comparison of velocity profile and tangential stress on the wall shows the NS's results have some difference with the kinetic results above the range of $\mathrm{Kn}$.

The new transport characters (mass, momentum and energy) make the gas-kinetic methods a more credible way. Theoretically, the direct simulation Monte Carlo (DSMC)

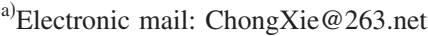

method can deal with broad flows; it is a statistical simulative technique proposed by Bird ${ }^{4}$ in 1969 . With some decades' development it has become an effective tool for many fields, including the rarefied gas effect, ${ }^{7}$ so it is feasible to use it for the study on microdimensional gas flows.

A nozzle is a basic device that offers thrust for the aerocraft system, and many small thrust nozzle systems have been used to maintain or adjust the orbit of space satellites. Since microsatellites appeared, they have needed a very fine control, which generally comes from small $(\mathrm{mm})$ or micro $(\mu \mathrm{m})$ nozzle systems. Therefore, the inner gas flows of micronozzles have become a focus, and it has been hoped that the understanding of the fluid characteristics could be helpful to the design of high performance. For the whole nozzle flows (in space), the gaseous fluids pass through all regimes, namely from continuum (chamber and convergent part of the nozzle) to free molecular flow (far from the exit of the nozzle), and it challenges the simulative capacity. In the 1960s, some papers appeared about small thrust (low Re number) nozzle flows, ${ }^{8-10}$ and so far people have accumulated much knowledge in this regard.

As for the experimental investigation, Rothe ${ }^{9}$ used electron-beam techniques to measure the flow density and rotational temperature at some inner and outer points of the nozzle, and visualized the external flow structure. Boyd et $a l .{ }^{11,12}$ used coherent anti-Stokes Raman scattering technique to measure the velocity and translational temperature of the selected positions of the nozzle, and at the same time measured the thrust of the nozzle. In particular, they simulated the whole flow field, and the agreement proved that the DSMC could be applied to small nozzle flows. Then Broc et $a l .{ }^{13}$ gained the temperature and density of outer jet flows with laser-induced fluorescence technique, and Jamison et $a l .{ }^{14}$ measured the thrust with and without a divergent part versus Reynolds number. Because of the difficulty in the measurement of the micronozzle, now we can only get some data about its total performance. Bayt et al. ${ }^{15}$ manufactured micronozzles with throat height of about $20-30 \mu \mathrm{m}$, and by testing they pointed out that the viscous resistance affected 


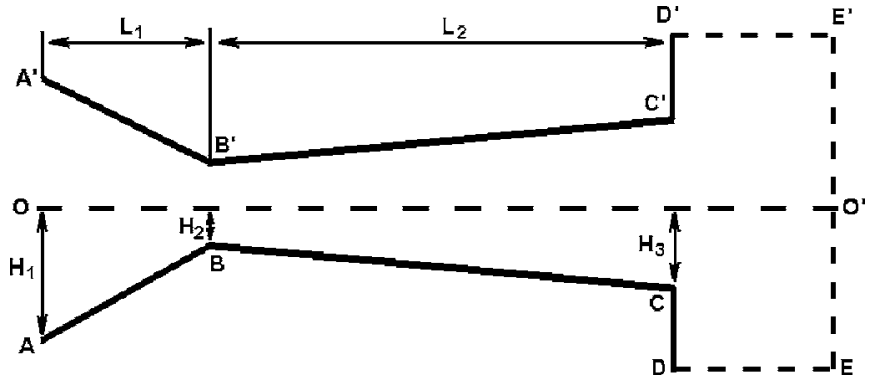

FIG. 1. The sketch of the simulated micronozzle.

flows mightily. Hao et al. ${ }^{16}$ also manufactured micronozzles (throat height $20 \mu \mathrm{m}$ ), and determined the dependence of the mass flux from the pressure difference (inlet's pressure minus back pressure) by keeping pressure at the inlet and decreasing the environmental pressure (back pressure) that connected with the outlet of the nozzle.

In comparison, more groups ${ }^{17-22}$ have investigated the nozzle flows or jet by computation. With DSMC, computational fluid dynamics (CFD), or coupled modeling, they have analyzed the flows in detail. Generally, the investigations are mainly focused on the thrust performance of nozzles working in the space. It is expected that the micronozzle system might be used in many fields under relatively higher backpressure conditions. ${ }^{23}$ So it is worthwhile to analyze the fluid characteristics under that condition.

In this paper, first the experimental cases are simulated. ${ }^{16}$ The gained mass flux is compared with data, and then the Mach numbers from DSMC and NS adding slip boundary results are compared. Under the condition of higher back pressure, the simulation gets the supersonic multiple expansion-compression waves phenomenon that issues from the divergent part of the nozzle. This is investigated in detail.

\section{SIMULATIVE CONDITIONS AND METHODS}

Figure 1 shows the shape of a plane two-dimensional micronozzle. The outline of the micronozzle is lined $A B C C^{\prime} B^{\prime} A^{\prime}$. The nozzle has three characteristic heights, i.e., throat $2 \mathrm{H}_{2}$, inlet $2 \mathrm{H}_{1}$, and outlet $2 \mathrm{H}_{3}$, respectively. $L_{1}$ is the length from the inlet section to the throat, and $L_{2}$ is the length from the throat to the outlet section. For the purpose of gaining correct results ${ }^{24}$ and the observation of the outer flow near the ports, a simulation region $C D E E^{\prime} D^{\prime} C^{\prime}$ is introduced for all the cases in this paper. The line $O O^{\prime}$ is the symmetrical line of the whole nozzle. It is easy to understand that the two-dimensional reduction is appropriate when the width-to-height ratio is a very large value. Actually, all the micronozzles (rectangular section) in experiments have a finite width. The error that comes from the above reduction has been analyzed carefully. ${ }^{17,19}$ The conclusion is that the mass flux would increase by about $3 \%$ from 3D to $2 \mathrm{D}$. The quoted experiment ${ }^{16}$ (throat height is $20 \mu \mathrm{m}$ and width is $120 \mu \mathrm{m})$ in the present paper has a similar dimension and width-to-height ratio to the above references. ${ }^{17,19}$ With consideration of the symmetry of the flow, only the subpart region, namely $O A B C D E O^{\prime}$, is simulated for the present cases. The mirror boundary condition is set to $O O^{\prime}$. For the walls $A B, B C$, and $C D$, two kinds of boundary conditions are tested. One is constant temperature $(300 \mathrm{~K})$, the other is an adiabatic wall. It has been found that the same results (relative error less than 1\%) are obtained for the different setting of the wall. Both of them are under the same diffuse reflection (Maxwell) condition. For the constant temperature wall, the accommodation coefficient (momentum and energy) is 1 . All figures in this paper are the results of the constant temperature wall. The main conditions can be found in Table I for every simulated case.

Initially the pressure is set at $A A^{\prime}$ and $D E E^{\prime} D^{\prime}$, respectively. Under the driving of the pressure difference, the

TABLE I. Conditions and coefficients for the present simulated cases. In all cases in this table, $H_{1}=34 \mu \mathrm{m}$, $H_{2}=10 \mu \mathrm{m}, L_{1}=50 \mu \mathrm{m}$, and $L_{2}=95 \mu \mathrm{m}$. The nondimensional coefficients $\mathrm{Kn}_{2}$ and $\operatorname{Re}_{2}$ are integral averaged Knudsen and Reynolds number calculated at the throat section, and $\mathrm{Kn}_{3}$ and $\mathrm{Re}_{3}$ are calculated at the outlet section. $\lambda_{\max }$ is the maximum local mean free path in the divergent part of the nozzle, and for all the present cases it appears only at point C (Fig. 1) or near the translational separate point along the wall of the divergent part. The symbol "-" means the flow has been separated and the average value at the outlet loses the worth of reference.

\begin{tabular}{lcccccccc}
\hline \hline $\begin{array}{l}\text { Case } \\
\text { no. }\end{array}$ & $\begin{array}{c}H_{3} \\
(\mu \mathrm{m})\end{array}$ & $\begin{array}{c}\text { Back } \\
\text { pressure } \\
P_{o}(\mathrm{kPa})\end{array}$ & $\begin{array}{c}\mathrm{Kn}_{2} \\
\left(\times 10^{-3}\right)\end{array}$ & $\begin{array}{c}\mathrm{Kn}_{3} \\
\left(\times 10^{-3}\right)\end{array}$ & $\begin{array}{c}\lambda_{\max } \\
(\mu \mathrm{m})\end{array}$ & $\mathrm{Re}_{2}$ & $\mathrm{Re}_{3}$ & $\begin{array}{c}\text { Correlate } \\
\text { with } \\
\text { picture no. }\end{array}$ \\
\hline 1 & 17 & 65 & 4.3 & 2.9 & 0.11 & 247 & 238 & Fig. 3(a) \\
2 & 17 & 55 & 4.4 & 3.4 & 0.14 & 260 & 253 & Fig. 3(b) \\
3 & 17 & 10 & 4.5 & 7.3 & 0.5 & 257 & 311 & Fig. 3(c) \\
4 & 17 & 30 & 4.4 & 5.4 & 0.23 & 259 & 277 & Fig. 4(a) \\
5 & 25 & 30 & 4.7 & - & 0.3 & 270 & - & Figs. 4(b) and 5 \\
6 & 35 & 30 & 4.8 & - & 0.32 & 276 & - & Figs. 4(c) and 4(d) \\
7 & 25 & 20 & 4.7 & - & 0.3 & 270 & - & Figs. 6(a) and 7(a) \\
8 & 25 & 10 & 4.7 & 7.6 & 0.7 & 269 & 335 & Figs. 6(b) and 7(b) \\
9 & 25 & 1 & 4.7 & 7.9 & 1.1 & 269 & 355 & Figs. 6(c) and 7(c) \\
\hline \hline
\end{tabular}




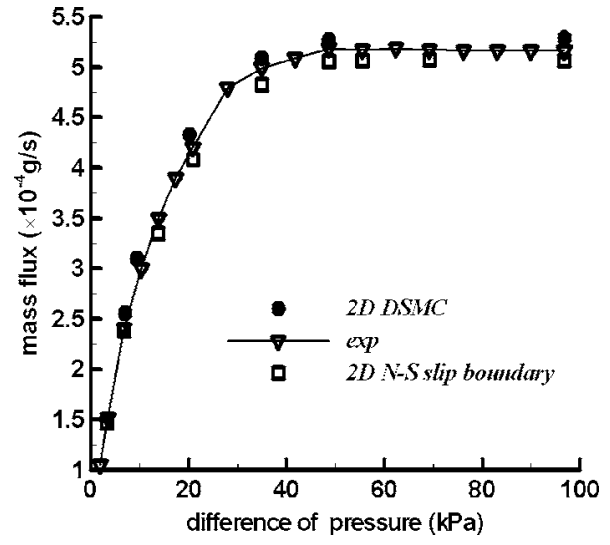

FIG. 2. Comparison of DSMC, NS, and experimental data for mass flux vs pressure difference.

nozzle builds up a flow field. It is presumed that the flows went through an isothermal process when it came from the chamber to the inlet section, so the total pressure at the inlet holds constantly at $1 \mathrm{~atm}$. The static temperature of the free flow is $300 \mathrm{~K}$. To the outflow boundary $D E E^{\prime} D^{\prime}$ the static pressure is set. With the appearance of supersonic flow at this boundary, the extrapolation is used to correct the physical profiles such as velocity, temperature, and density in the progress of calculative convergence. ${ }^{24-26}$ The work gas is air (78.5\% nitrogen and $21.5 \%$ oxygen).

The DSMC's code is a self-developed 2D edition named "plane-2004." It has higher power to deal with complicated geometry of microdevices. Many previous cases have tested its reliability. Like many other DSMC codes, this edition divides the flow field into many small cells, and maintains their characteristic dimension about the order of the local mean molecular free path. The subcell technique ${ }^{4}$ has also been used to limit the collision of molecular pairs within half of the free path. Agreeing with the above rules, the time step is smaller than the local mean collision time. The $\mathrm{NTC}^{4}$ collision selection method is applied. In addition to the basic rules about the DSMC method, this edition has some optimized skills added that come from the author's experience of simulation in rarefied gas dynamics (RGD). The finite volume method ${ }^{33}$ is used to calculate the compressible real gas NS equations. The first-order slip boundary condition is also used, and its mesh is similar to that of DSMC.

\section{RESULTS AND ANALYSES}

Figure 2 shows the dependence of the mass flux on the pressure difference between the DSMC, the experimental data, and NS's results. It evidently shows the evolutional process from stillness to the choked state. The good agreement is shown. Quantitatively, the DSMC result is larger than the experimental data, about $2 \%-4 \%$ in the whole range of pressure difference, and the NS result is about 1\%-3\% smaller than the experimental data. For the reason explained in the text "simulative conditions and methods" (namely cut down by $3 \%$ from $2 \mathrm{D}$ to $3 \mathrm{D}$ ), finally in the region of the choked state, the corrected relative error is about $1 \%$ between DSMC results and the experimental data, and $5 \%$ be-

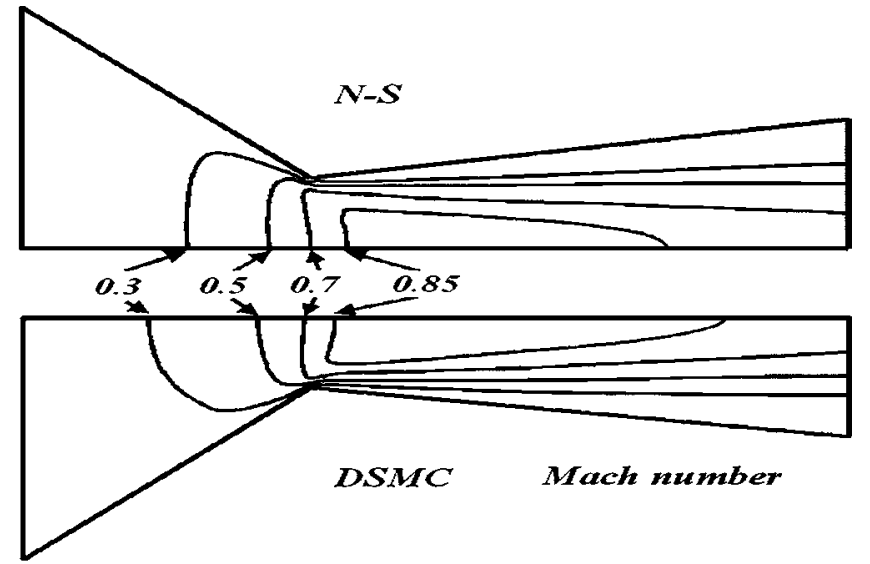

(a)

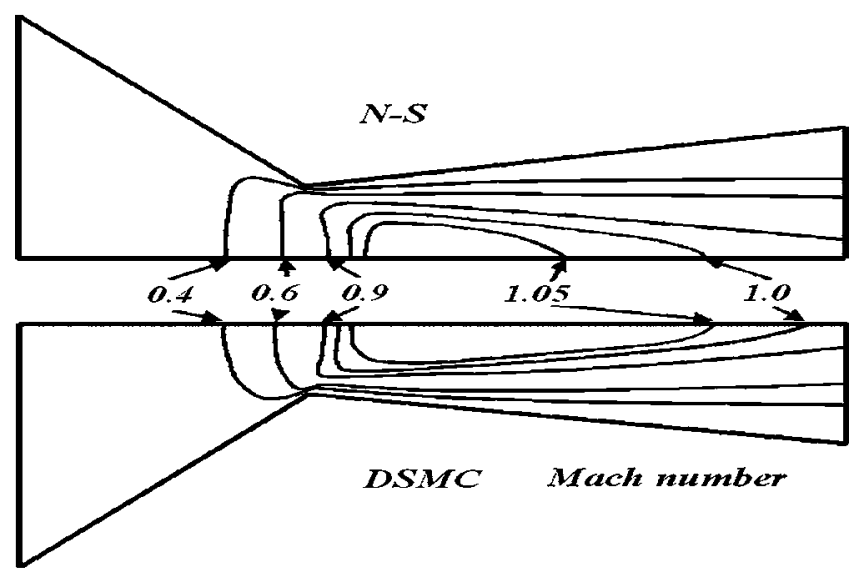

(b)

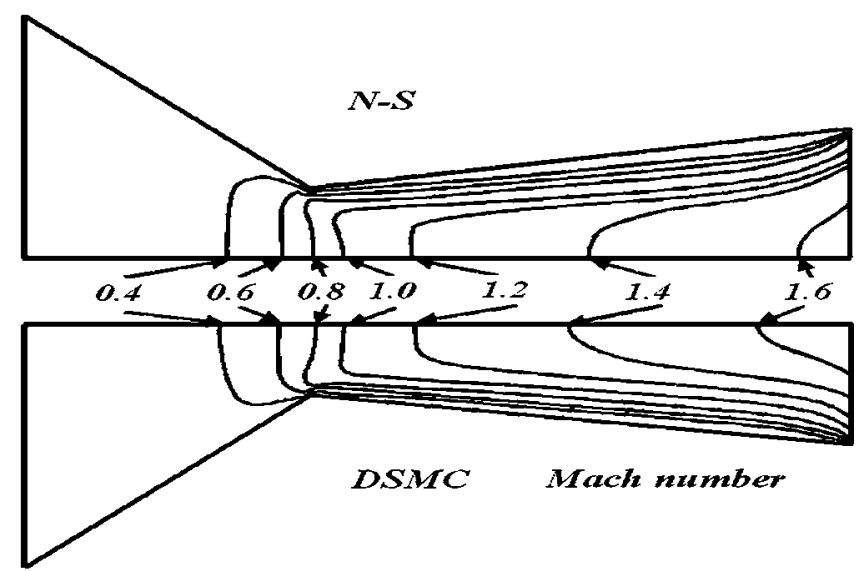

(c)

FIG. 3. Comparison of DSMC and NS for Mach numbers under three backpressure conditions. (a) Comparison of NS and DSMC for Mach number (case 1). (b) Comparison of NS and DSMC for Mach number (case 2). (c) Comparison of NS and DSMC for Mach number (case 3).

tween NS results and the experimental data, respectively. The uncertainty of the measurement is less than $2 \%{ }^{16}$

For the investigation of inner flows, Fig. 3 compares Mach number contours gained by NS (upper) and DSMC (lower) under three back-pressure conditions: 65, 55, and $10 \mathrm{kPa}$, respectively. It is different from general knowledge about supersonic nozzle flows (higher Re number flow) that the pressure difference of $36 \mathrm{kPa}$ [Fig. 3(a)] cannot build the 


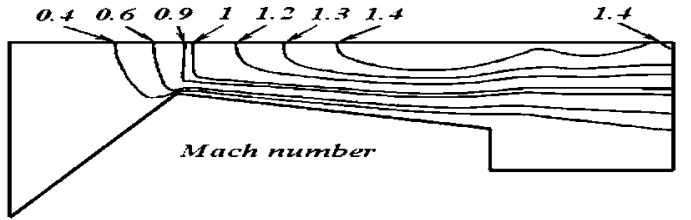

(a)

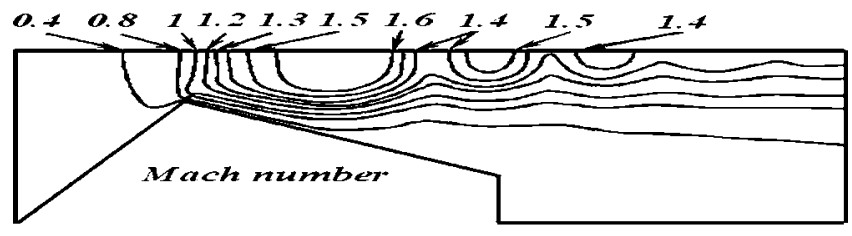

(b)

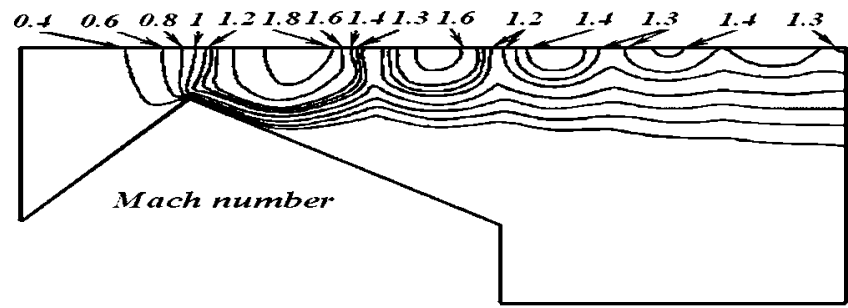

(c)

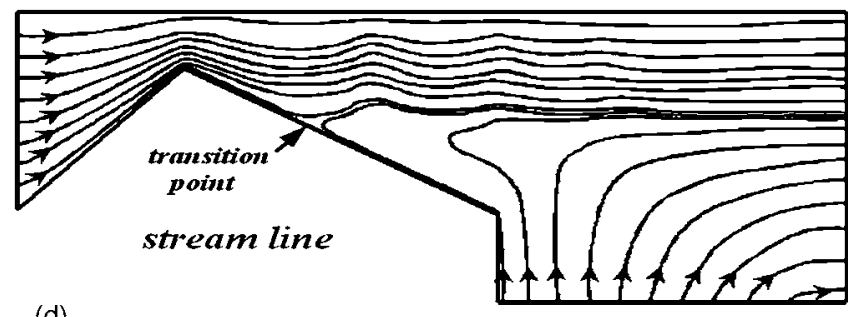

(d)

FIG. 4. Comparison of Mach numbers with three different outlets' heights $\mathrm{H}_{3}$ and the show of separate phenomena. (a) Mach number for case 4. (b) Mach number for case 5. (c) Mach number for case 6. (d) Stream line for case 6.

supersonic flow in the divergent part of the nozzle. It shows a high-speed region behind the throat $(\mathrm{Ma}=0.7)$. Similar trends come from the two methods, and the DSMC obtained a larger high Mach number region. The sonic line (Ma $=1.0$ ) appears behind the throat section when the back pressure falls to $55 \mathrm{kPa}$ [Fig. 3(b)]. Passing through the sonic line, the flow is accelerated to a supersonic speed, but then it falls unexpectedly to the subsonic state. It shows a subsonic profile at the outlet of the nozzle. It is similar to the first case [Fig. 3(a)] that the DSMC gains a larger high Mach number region again. When the back pressure is reduced to $10 \mathrm{kPa}$ [Fig. 3(c)], the flow fully enters the choked state. The gas passes through the whole nozzle with continuous acceleration. Although the relative difference between DSMC and NS decreases, the DSMC gains a larger Mach number near the outlet. As for the DSMC, the maximal Mach numbers for the above three cases are about $0.96,1.17$, and 1.74 . When it comes to the NS, the numbers are about $0.92,1.12$, and 1.69.

Table I lists the average $\mathrm{Kn}$ and Re number. They are calculated at the throat and the outlet sections, respectively, and the variables temperature $T$ and pressure $P$ are the averaged integral values along the two sections, respectively:

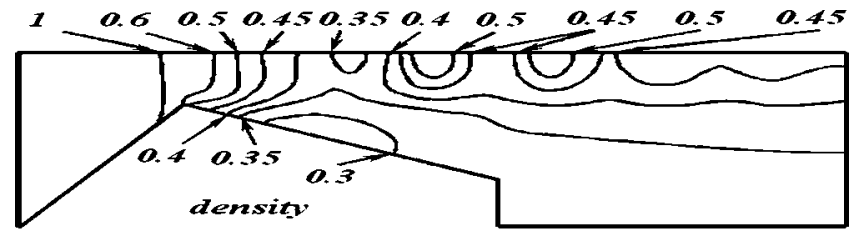

(a)

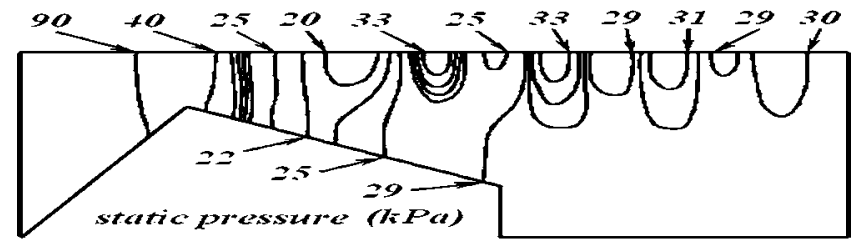

(b)

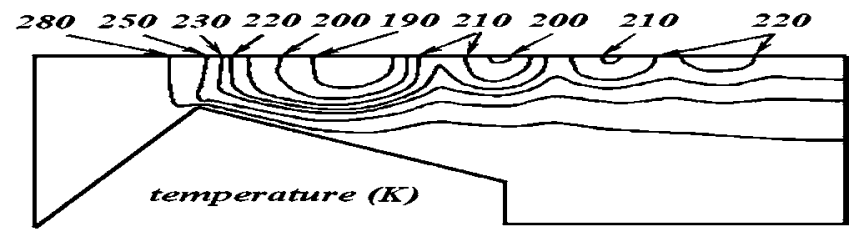

(c)

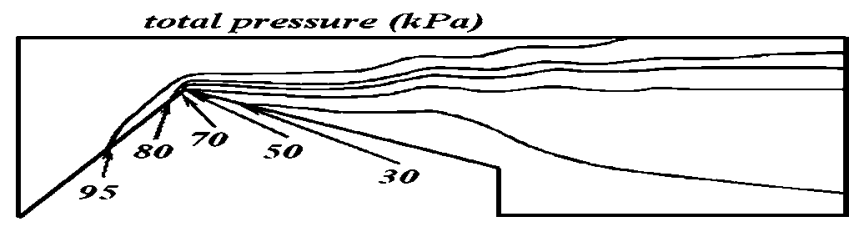

(d)

FIG. 5. Variable contours for one case in which back pressure $P_{o}=30$ and $30 \mathrm{kPa}$ and $H_{3}=25 \mu \mathrm{m}$. (a) Mass density contour (unit $\mathrm{kg} / \mathrm{m}^{3}$ ). (b) Static pressure contour (unit $\mathrm{kPa}$ ). (c) Translational temperature contour (unit K). (d) Total pressure contour (unit $\mathrm{kPa}$ ).

$$
\begin{aligned}
& \mathrm{Kn}=\frac{\lambda}{H}, \\
& \mathrm{Re}=\frac{U H \rho}{\mu}=\frac{\dot{m}}{\mu} .
\end{aligned}
$$

The symbol $\dot{m}$ is the mass flux of unit width, and the viscous coefficient $\mu=\mu(T)$ employs the Sutherland relation. The mean molecular free path is defined by the formulation

$$
\lambda=\frac{16}{5} \sqrt{\frac{k T}{2 \pi m}} \frac{\mu}{P},
$$

where $k$ is the Boltzmann constant and $m$ is the molecular mass. The characteristic height $\mathrm{H}$ at two sections is $2 \mathrm{H}_{2}$ and $2 \mathrm{H}_{3}$, respectively.

From the coefficients listed in Table I, we can see that even if the average $\mathrm{Kn}$ number is smaller than 0.01 , there is still deflection between NS and DSMC results for the above cases.

Actually, one more correct judgement of rarefied gas effect may be based on the local breakdown parameter ${ }^{4}$ 


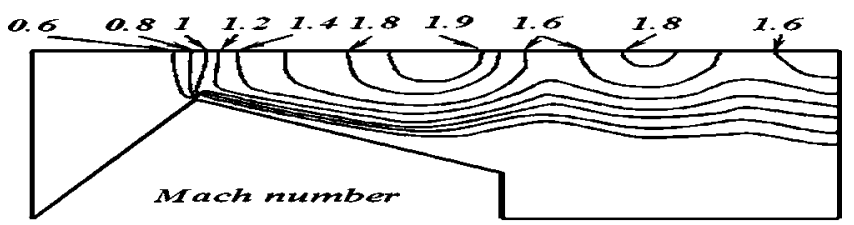

(a)

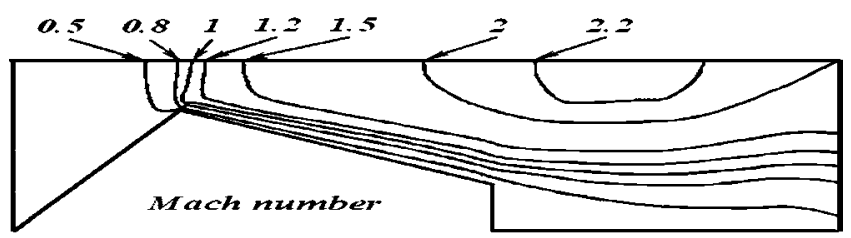

(b)

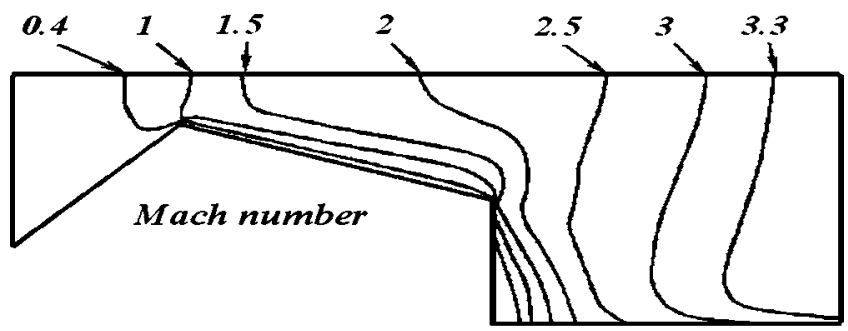

(c)

$$
P_{\text {break }} \approx \frac{\lambda_{\text {local }}}{\Re}\left|\frac{d \Re}{d s}\right|,
$$

where the $\lambda_{\text {local }}$ is the local mean molecular free path, $\mathfrak{R}$ is a local physical variable, and $s$ is the distance along the streamline. The higher parameter value means the greater invalidity of the continuum model. In some part of the flow field, this parameter may step into the critical value earlier. This parameter may also partly explain the increasing trend of the difference between the experimental data and the NS result with the evolution of the choked state. It is the higher speed that causes a more tempestuous change of variables in the flow field, and then a higher local $P_{\text {break }}$ value appears.

By changing the expansion ratios $\left(\mathrm{H}_{3} / \mathrm{H}_{2}\right)$, the nozzle can obtain a new fluid pattern and performance. In the following paragraphs, we will analyze the transformation of the pattern with expansion ratios and pressure difference.

First of all, the back pressure is fixed to $30 \mathrm{kPa}$. With the changing of the outlet height $\mathrm{H}_{3}$, a series of cases are shown in Fig. 4. Figures 4(a)-4(c) show Mach number contours for different $\mathrm{H}_{3}$. We can find in them that the novel supersonic "multiwaves" phenomenon occurs on the symmetrical line $O O^{\prime}$, which shows the fluctuant Mach number. With the heightening of $\mathrm{H}_{3}$ [Fig. 4(b)], the "multiwaves" become evident, and the intensity of the waves (relative change between peak and trough values of every wave along line $O O^{\prime}$ ) increases. At the same time, the maximal Mach number in the first wave's core increases from 1.53 [Fig. 4(a)] to 1.79 [Fig. 4(b)]. In every case, the multiwaves take on a lessened trend, i.e., the intensity of the latter one is smaller than the foregoing one. This trend becomes very evident when $H_{3}$ is heightened to $35 \mu \mathrm{m}$ [Fig. 4(c)]. The comparison of the above three cases shows that the first wave moves to the throat, and the sonic line tends toward the throat section with the increase of $\mathrm{H}_{3}$. An important flow separation is found for Figs.
FIG. 6. Evolution of Mach number with the decreasing of back pressure, $H_{3}=25 \mu \mathrm{m}$. (a) Mach number contour for case 7. (b) Mach number contour for case 8. (c) Mach number contour for case 9.

4(b) and 4(c). Figure 4(d) shows that the streamlines correspond to Fig. 4(c). It shows the environmental gas near the outlet has been induced by a supersonic jet, and the induced gas backwashed into the inner divergent part of the nozzle and then encountered the main flow at the transitional point. This means that there is backward pressure grads to drive the backflows.

With the detailed analyses of flow fields, we can reveal the essence of the "multiwaves." Figure 5 shows some physical variable contours that are the same case as Fig. 4(b). Figure $5(\mathrm{a})$ is the mass density contour (unit $\mathrm{kg} / \mathrm{m}^{3}$ ). It shows that the density decreases gradually from the inlet to the throat, and behind the throat it arrives at about 0.35 , the local minimal value, and then it shows a similar wave structure to the Mach number contour. As for Fig. 5(b), the static pressure contour shows a low-pressure region behind the throat, and it also has a wave structure. Figure 5(c) shows the translational temperature. Figure 5(d) shows the total pressure, based upon which we can observe the loss of the total pressure and the growing of the boundary layer. It proves the existence of strong viscous dissipation and the boundary layer affects the main region of the divergent part of the nozzle. Summing up the above physical variable fields, we can conclude that the so-called "multiwaves" are a supersonic expansion-compression waves structure, which occurs after the overexpanded jet and falls into a decline by dissipation. In spite of the intense shear stress near the wall, we can still find a nonviscous core ${ }^{23}$ in the jet's center region near the throat.

In fact, a similar phenomenon has been partly found $^{9,13,27}$ and investigated (please see Fig. 9 in Ref. 9) in the field of rarefied gas dynamics. Generally, one of the following running states may appear ${ }^{23,28,29}$ when the nozzle's flow is with high Re number: if the environmental back pres- 


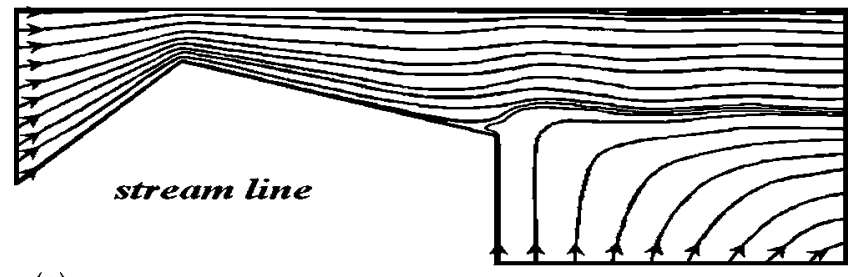

(a)

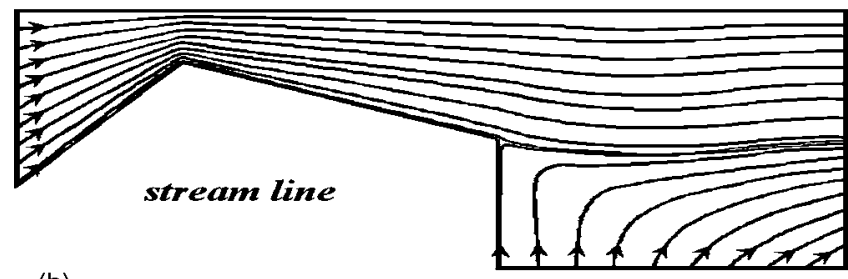

(b)

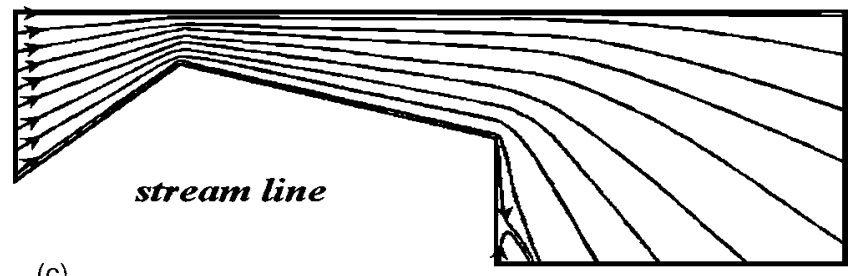

(c)

FIG. 7. Evolution of streamline with decreasing of back pressure, $\mathrm{H}_{3}$ $=25 \mu \mathrm{m}$. (a) Streamline for case 7. (b) Streamline for case 8. (c) Streamline for case 9 .

sure is much smaller than the static pressure of the expanded gas that just goes out from the outlet, the outflow will be accelerated continuously and pass through an underexpanded process. If the back pressure is not so small, the gas will pass by the outlet with an overexpanded process, and then it will be enveloped by a barrel-shaped shock wave structure. The excessively compressed gas is followed by an overexpanded process, which may be repeated damply to make the multiwaves. The strong right shock wave corresponds to a high ratio of environmental pressure to the static pressure of expanded gas. When the ratio is big enough, the shock wave will move toward the inner nozzle. For rarefied gas nozzle flows with low Re number, the thickened boundary layer and the rapid loss of kinetic energy make it easier to separate the main flow at the wall of its divergent part. Then the environmental gas moves to the transitional point and compresses the main flow that has been overexpanded, so the multiwaves appear in the divergent part of the nozzle. With the similar relationship (Knudsen number, Reynolds number, and Mach number) between the small nozzle ${ }^{9}$ and the MEMS's micronozzle, the above phenomenon occurs under some pressure conditions. Furthermore, the separation becomes more possible when the microthroat of the nozzle remarkably decreases the pressure. ${ }^{30-32}$

Finally, we observe the dependence of the fluid pattern on the back pressure for a fixed geometry. Figures 4(b) and 6 show a series of Mach numbers; the $H_{3}=25 \mu \mathrm{m}$ and the back pressure decreases from 30 to $1 \mathrm{kPa}$. Figure 7 shows three pictures of streamline that correspond to the Mach numbers in Fig. 6. With the pictures we can see the multiwaves move toward the nozzle's outer part with the decrease of back pressure, and the separation disappears when the back pressure is $10 \mathrm{kPa}$ [Fig. 6(b)]. The small change within the nozzles [Figs. 6(b) and 6(c)] reveals that the flows have stepped into the choked state.

\section{CONCLUSIONS}

The comparison between the DSMC's results and the experimental data shows that the statistical method can deal well with the near-sonic flows in the MEMS. The discrepancy (about 5\%) between NS equations adding a slip boundary condition and the experimental data shows that the continuum assumption gradually becomes invalid; even the average Kn number is smaller than 0.01 for the present cases. The larger loss of pressure comes from the throat, and the wall's boundary layer makes it easier to form the supersonic multiple expansion-compression waves in the nozzle's divergent part. This is a factor that may affect the nozzle's performance when it works under the condition of lower-pressure difference. The DSMC method clearly depicts the variable fields.

${ }^{1}$ G. E. Karniadakis and A. Beskok, Micro Flows (Springer, Berlin, 2001).

${ }^{2}$ S. A. Schaaf and P. L. Chambre, Flow of Rarefied Gases (Princeton University Press, Princeton, 1958).

${ }^{3}$ V. P. Shidlovskiy, Introduction to Dynamics of Rarefied Gases (Elsevier, Amsterdam, 1967).

${ }^{4}$ G. A. Bird, Molecular Gas Dynamics and the Direct Simulation of Gas Flow (Clarendon, New York, 1994).

${ }^{5} \mathrm{C}$. Cercignan, The Boltzmann Equation and Its Applications (SpringerVerlag, Berlin, 1988).

${ }^{6}$ N. G. Hadjiconstantinou, "Comment on Cercignani's second-order slip coefficient," Phys. Fluids 15, 2352 (2003).

${ }^{7}$ E. S. Oran, C. K. Oh, and B. Z. Cybyk, "Direct simulation Monte Carlo: Recent advances and applications," Annu. Rev. Fluid Mech. 30, 579 (1998).

${ }^{8} \mathrm{M}$. W. Milligan, "Nozzle characteristics in the transition regime between continuum and free molecular flow," AIAA J. 2, 1088 (1964).

${ }^{9}$ D. E. Rothe, "Electron-beam studies of viscous flow in supersonic nozzles," AIAA J. 9, 804 (1971).

${ }^{10}$ W. J. Rae, "Some numerical results on viscous low-density nozzle flows in the slender-channel approximation," AIAA J. 9, 811 (1971).

${ }^{11}$ I. D. Boyd, P. F. Penko, D. L. Meissner, and K. J. Dewitt, "Experimental and numerical investigations of low-density nozzle and plume flows of nitrogen," AIAA J. 30, 2453 (1992).

${ }^{12}$ I. D. Boyd and D. B. VanGilder, "Computational and experimental investigations of rarefied flows in small nozzles," AIAA J. 34, 2320 (1996).

${ }^{13}$ A. Broc, S. D. Benedictis, G. Dilecce, M. Viglotti, R. G. Sharafutdinov, and P. A. Skovorodko, "Experimental and numerical investigation of an O2/NO supersonic free jet expansion," J. Fluid Mech. 500, 211 (2004).

${ }^{14}$ A. D. Jamison and A. D. Ketsdever, "Low Reynolds number performance of an underexpanded orifice and a Delaval nozzle," in Rarefied Gas Dynamics $23 r$, edited by A. D. Ketsdever and E. P. Muntz (AIP, New York, 2003).

${ }^{15} \mathrm{R}$. Bayt, "Analysis, fabrication and testing of a MEMS-based micropropulsion system," Ph.D. thesis, MIT (1999).

${ }^{16}$ P. F. Hao, Y. T. Ding, Z. H. Yao, F. He, and K. Q. Zhu, "Size effect on gas flow in micro nozzles," J. Micromech. Microeng. 15, 2069 (2005).

${ }^{17}$ A. A. Alexeenko, R. J. Collins, S. F. Gimelshein, and D. A. Levin, "Challenges of three-dimensional modeling of microscale propulsion devices with the DSMC method," in Rarefied Gas Dynamics 22nd, edited by T. J. Bartel and M. A. Gallis (AIP, New York, 2000).

${ }^{18}$ A. A. Alexeenko, D. A. Fedosov, S. F. Gimelshein, D. A. Levin, and R. J. Collins, "Transient heat transfer and gas flow in a MEMS-based thruster," J. Microelectromech. Syst. 15, 181 (2006). 
${ }^{19}$ G. N. Markelov and M. S. Ivanov, "Numerical study of 2D/3D micronozzle flows," in Rarefied Gas Dynamics 22nd, edited by T. J. Bartel and M. A. Gallis (AIP, New York, 2000).

${ }^{20}$ E. V. Titov and D. A. Levin, "Application of the DSMC and NS techniques to the modeling of a dense, low Reynolds number MEMS device," in Rarefied Gas Dynamics 24th, edited by M. Capitelli (AIP, New York, 2004).

${ }^{21} \mathrm{M}$. Usami and K. Teshima, "Molecular simulation of rarefied supersonic free jets by DSMC method," JSME Int. J., Ser. B 42, 369 (1999).

${ }^{22} \mathrm{M}$. Usami and K. Teshima, "Three dimensional simulation on deformation of jet boundary in an underexpanded axisymmetric jet," in Rarefied Gas Dynamics 24th, edited by M. Capitelli (AIP, New York, 2004).

${ }^{23}$ A. E. Beylich, "Structure and applications of jets," in Rarefied Gas Dynamics 21st, edited by R. Brun, R. Campargue, R. Gatignol, and J.-C. Lengrand (CEPADUES EDITIONS, Marseille, France, 1998).

${ }^{24}$ C. Xie, "Subsonic choked flow in micro-channel," Phys. Fluids 18, 127104 (2006).

${ }^{25}$ C. Xie, J. Fan, and C. Shen, "Statistical simulation of rarefied gas flows in micro-channels," in Rarefied Gas Dynamics 23rd, edited by A. D. Ketsdever and E. P. Muntz (AIP, New York, 2003).

${ }^{26}$ C. Shen, J. Fan, and C. Xie, "Statistical simulation of rarefied gas flows in micro-channels," J. Comput. Phys. 189, 512 (2003).

${ }^{27}$ T. Hyakutake and K. Yamamota, "Numerical simulation of rarefied plume flow exhausting from a small nozzle," in Rarefied Gas Dynamics 23rd, edited by A. D. Ketsdever and E. P. Muntz (AIP, New York, 2003).

${ }^{28}$ P. Vankan, S. Mazouffre, R. Engeln, and D. C. Schram, "Inflow and shock formation in supersonic, rarefied plasma expansions," Phys. Plasmas 12, 102303 (2005).

${ }^{29}$ G. P. Sutton and O. Biblarz, Rocket Propulsion Element (Wiley, New York, 2001).

${ }^{30}$ I. G. Khalil and D. R. Miller, "The free-jet expansion of supercritical CO2 from a capillary source," in Rarefied Gas Dynamics 24th, edited by M. Capitelli (AIP, New York, 2004).

${ }^{31}$ C. Raju and J. Kurian, "Experimental investigation of rarefied gas flow through rectangular slits and nozzles," Exp. Fluids 17, 220 (1994).

${ }^{32}$ F. Sharipov, "Numerical simulation of rarefied gas flow through a thin orifice," J. Fluid Mech. 518, 35 (2004)

${ }^{33}$ J. R. Patrick, Computational Fluid Dynamics (Hermosa, New York, 1972). 